\title{
Reconstruction of the Shapes of Gold Nanocrystals Using Coherent X-Ray Diffraction
}

\author{
I. K. Robinson, I. A. Vartanyants,* G. J. Williams, M. A. Pfeifer, J. A. Pitney ${ }^{\dagger}$ \\ Department of Physics, University of Illinois, Urbana, Illinois 61801
}

(Received 15 May 2001; published 19 October 2001)

\begin{abstract}
Inverse problems arise frequently in physics: The magnitude of the Fourier transform of some function is measurable, but not its phase. The "phase problem" in crystallography arises because the number of discrete measurements (Bragg peak intensities) is only half the number of unknowns (electron density points in space). Sayre first proposed that oversampling of diffraction data should allow a solution, and this has recently been demonstrated. Here we report the successful phasing of an oversampled hard x-ray diffraction pattern measured from a single nanocrystal of gold.
\end{abstract}

DOI: $10.1103 /$ PhysRevLett.87.195505

Because of the unsurpassed power of crystallography to reveal the structure of matter at the atomic scale [1], a number of important methods have been proposed for avoiding its inherent phase problem. Generally speaking, these work by adding supplementary information, such as statistical chemical knowledge [2], solvent flatness [3], noncrystallographic symmetry [4], or "maximum entropy" of the solution [5]. The utility of oversampling diffraction patterns was considered by Sayre [6] and developed as a practical method, which has since been proven computationally $[7,8]$. At least twofold oversampling of the data is required, in order to exceed the spatial Nyquist frequency, whereby the inversion becomes mathematically overdetermined. Oversampling is not possible for diffraction from macroscopic crystals because all the intensity is concentrated in Bragg peaks. It has been demonstrated computationally $[7,8]$, however, that inversions are possible for inherently nonperiodic objects whose diffraction patterns are continuous functions. Recently, Miao et al. used oversampling to invert the soft $\mathrm{x}$-ray forward-scattering pattern measured from a fabricated object [9].

The oversampling approach is different from, but related to, holographic methods [10]. In holography, a known "reference wave" is caused to interfere with a wave of unknown phase so that the phase is revealed as intensity variations. Applications have been developed to probe local structure by photoelectron diffraction $[11,12]$ and x-ray fluorescence imaging [13-15]. Three-beam diffraction phasing methods for crystallography [16], mirror-based heterostructure phase determination [17], and phasesensitive tomography [18] are also based on the concept of a reference wave.

Our experiments extend the previous successes with oversampling as an inversion tool [7-9] to the case of diffraction from single crystals, sufficiently small to generate a continuous diffraction pattern, which can be oversampled. This requires the coherence length of the $\mathrm{x}$-ray beam to be bigger than the sample, in the range of a few microns, as is now achievable with the latest sources of synchrotron radiation. When a crystal of nanometer dimensions is illuminated by such a beam, its diffraction is no longer comprised of sharp Bragg peaks, but of a com-
PACS numbers: 61.10.Dp, 42.30.Rx, 68.35.Md, 81.07.Bc

plicated intensity distribution centered at each reciprocal lattice point [19]. For an ensemble of many particles, the width of the average intensity distribution is just given by the "finite-size effect." A single particle gives a detailed distribution instead, which can be measured on a sufficiently fine scale to fulfill the oversampling requirement.

Inversion of such a diffraction pattern is an overdetermined analytical problem [20]. The solution can be reached by a number of iterative methods based on the procedure proposed by Gerchberg and Saxton (GS) [21] and since developed further by Fienup [22] and others. There is a formal analogy, pointed out by Millane [23], between these image-seeking methods and the aforementioned "density modification" methods in crystallography [3-5]. Known amplitudes are combined with random phases and Fourier transformed to make an initial estimate of the real-space structure. This is then updated by applying a real-space constraint and backtransformed. The phases generated are combined with the measured amplitudes and the cycle is repeated. We will show in this paper that the method can be used to invert an x-ray diffraction pattern into an image of the nanocrystal from which it originates.

The gold nanocrystals examined in these experiments were prepared by heating thin films in a furnace at $950{ }^{\circ} \mathrm{C}$ for around $100 \mathrm{~h}$. The films were prepared by evaporation of gold onto silicon wafers, used as $\mathrm{SiO}_{2}$ substrates, to a thickness of $1000 \AA$. During heating, the uniform films first break up into one-dimensional ribbons, then into individual droplets with approximately uniform separation, as monitored by scanning-electron microscopy (SEM) and in good agreement with the findings of Heyraud and Métois [24]. These droplets, once cooled to room temperature, were found to be single crystals with faceted morphologies, probably related to the equilibrium crystal shape at the annealing temperature [25]. The resulting crystals had a fairly narrow size distribution centered around $1 \mu \mathrm{m}$ diameter. Similar treatment of $3000 \AA$ A thick films was found to produce crystals around $4 \mu \mathrm{m}$ in diameter.

The nanocrystals were measured at Sector 33 of the Advanced Photon Source (APS) using undulator radiation of $1.65 \AA$ wavelength. The beam was prepared with a double 
crystal $\mathrm{Si}(111)$ monochromator, without focusing, and filtered by reflection from two Pt-coated mirrors. The lateral coherence length of around $5 \mu \mathrm{m}$, given by the source size [26], and the longitudinal coherence length of around $0.3 \mu \mathrm{m}$, given by the monochromator bandwidth, were both sufficient for the measurements. Slits in front of the sample were used to produce a $30 \mu \mathrm{m} \times 100 \mu \mathrm{m}$ footprint on the sample at the center of the diffractometer. The nanocrystals were found to have $\{111\}$ texture with random orientation about the substrate surface normal. By

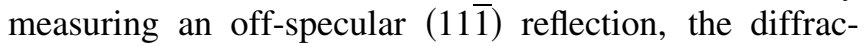
tion from each of the 300 crystals within the illuminated area could be separated by scanning the diffractometer's $\phi$ axis. Approximately 300000 counts per second were recorded from each $1 \mu \mathrm{m}$ crystal, integrated over a wide

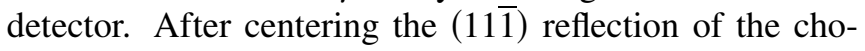
sen crystal, the detector was replaced with a direct-reading charge-coupled device (CCD), with $22.5 \mu \mathrm{m} \times 22.5 \mu \mathrm{m}$ pixels, situated $2.8 \mathrm{~m}$ from the sample, giving a resolution of $\Delta q=3 \times 10^{-5} \AA^{-1}$ per pixel in both the vertical and the horizontal directions. Multiple accumulations of the diffraction pattern avoided saturation of the CCD and a beamstop was used to block out the intense central region, typically resulting in the pattern of Fig. 1.

All the $(11 \overline{1})$ coherent diffraction patterns we observed showed the two characteristic features seen in Fig. 1: the radial flares of intensity and the fringes of modulation along the flares. The diffraction patterns obtained at the exact (11) Bragg condition were found to be almost rotationally symmetric about their apparent center, as seen in Fig. 1. This symmetry was quickly lost when the Bragg angle, $\omega$, was moved away from center in steps of $0.001^{\circ}$ : The flare on one side of the pattern increased in intensity at the expense of the one on the opposite side. This local symmetry can be understood from the following considerations.

If the atoms of the crystal lie on a perfect lattice, $L(\mathbf{r})$, bounded by some shape, $\Gamma$, the diffracted intensity will be given by the square magnitude of a complex amplitude,

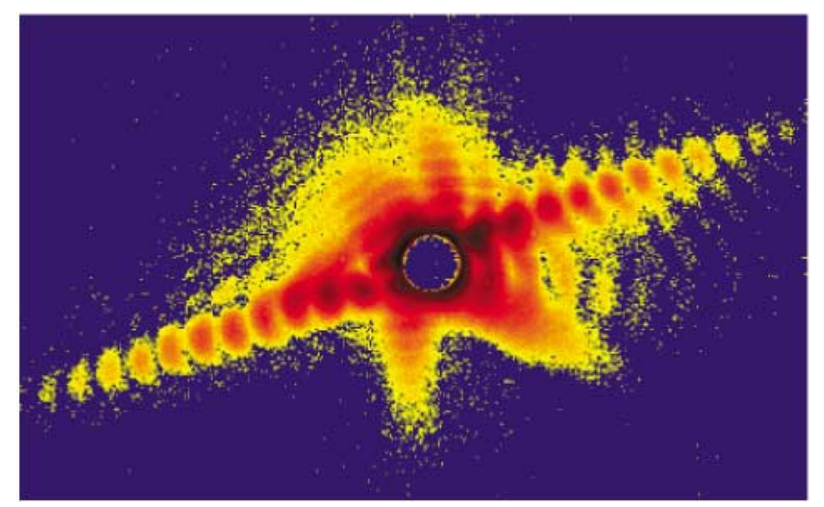

FIG. 1 (color). Coherent $x$-ray diffraction pattern of the immediate vicinity of the (111) Bragg reflection of a $1 \mu \mathrm{m}$ gold nanocrystal. A beamstop blocks the center. The color contour levels are logarithmically spaced.
$A(\mathbf{q})$, expressed as a function of total momentum transfer, $\mathbf{q}$, in reciprocal space. $A(\mathbf{q})$ is proportional to the Fourier transform of the electron density everywhere inside the crystal:

$$
\begin{gathered}
A(\mathbf{q})=\int s_{\Gamma}(\mathbf{r}) L(\mathbf{r}) \exp (i \mathbf{q} \cdot \mathbf{r}) d \mathbf{r}, \\
s_{\Gamma}(\mathbf{r})=\left\{\begin{array}{l}
0: \mathbf{r} \notin \Gamma \\
1: \mathbf{r} \in \Gamma,
\end{array}\right. \\
L(\mathbf{r})=\sum_{n_{j}=-\infty}^{\infty} \delta\left(\mathbf{r}-n_{1} \mathbf{a}_{1}-n_{2} \mathbf{a}_{2}-n_{3} \mathbf{a}_{3}\right),
\end{gathered}
$$

where $\mathbf{a}_{j}$ are the crystal lattice vectors. Applying the convolution theorem to the product in Eq. (1) and noting that the Fourier transform of the lattice function, $L(\mathbf{r})$, is itself a lattice function in reciprocal space, we find that the Fourier transform of the shape function, $s_{\Gamma}(\mathbf{r})$, becomes repeated around every reciprocal lattice point, denoted $\mathbf{q}=\mathbf{h}$. The combination of this periodic symmetry, $A(\mathbf{h}+\mathbf{q})=A(\mathbf{q})$, with the centrosymmetry of Eq. (1), $A(\mathbf{q})=A^{*}(-\mathbf{q})$, gives the local symmetry we observed in the data: $A(\mathbf{h}+\delta \mathbf{q})=A^{*}(\mathbf{h}-\delta \mathbf{q})$. Empirically, we can identify the Bragg point, $\mathbf{q}=\mathbf{h}$, in three dimensions (3D) with the rotation center (2D) of the most symmetric diffraction pattern of the $\omega$ series (1D).

The deviations from local symmetry are attributed to crystal imperfections which result in strains. Strain in the crystal will cause its atoms to move from exact lattice sites, so Eq. (3) will no longer apply. It can be shown [27] that this can be approximated by allowing the shape function, $s_{\Gamma}(\mathbf{r})$, to become complex, with an imaginary part proportional to the displacement projected onto the direction of q. Since a complex valued object no longer has a centrosymmetric diffraction pattern, the local symmetry is lost.

The flares point in the directions of the surface normals of the nanocrystal's facets. The strongest flare, bottom

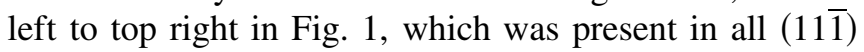
images, is close to the substrate surface normal, which is the crystal's (111) direction. The enhancement of intensity along the direction of facets is a well-known diffraction effect anticipated by von Laue [28] by expressing the volume integral for the diffraction of a small object as a surface integral and noting its singular directions. This is also the origin of the crystal truncation rods [29] widely studied in diffraction from surfaces. The fringes are a coherent diffraction effect that is most pronounced when two facets are parallel to each other, causing strong interference. We performed test calculations of the 3D Fourier transform of polyhedral objects and found flares both with and without fringes, depending on whether facets were paired. Sections of the calculated transform could be made to closely resemble the data by a suitable choice of facets.

We have thus demonstrated that the diffraction pattern surrounding every Bragg point is given by the square 
modulus of the Fourier transform of the shape function, $s_{\Gamma}(\mathbf{r})$, describing the external shape of the nanocrystals. For an unstrained crystal, the pattern surrounding a Bragg peak is the same as that surrounding the origin of reciprocal space. However, there are four important advantages to not using the forward scattering data near $\mathbf{q}=0$ : (i) If each grain in the sample has a different orientation, their patterns are superimposed at the origin, but separated at the Bragg peaks. (ii) Contaminating signals from all other objects in the beam (including air background) will be present around the origin. (iii) The direct beam obscures the center of the pattern, which then has to be synthesized [9]. (iv) The external shape of the particle, as seen by SEM, for example, is not necessarily the same as the boundary of the crystalline core, since it may have a "skin" of disordered material.

We therefore sought to invert the diffraction pattern to obtain a $2 \mathrm{D}$ real-space image of the shape, $\Gamma(\mathbf{r})$, projected in the direction of the exit wave vector. We used data from the (111) specular reflection shown in Fig. 2(a), for which the oversampling requirement is amply fulfilled, since each 2D fringe contains over 100 pixels. To define the origin of reciprocal space, $\mathbf{q}=\mathbf{h}$, we located the cen-
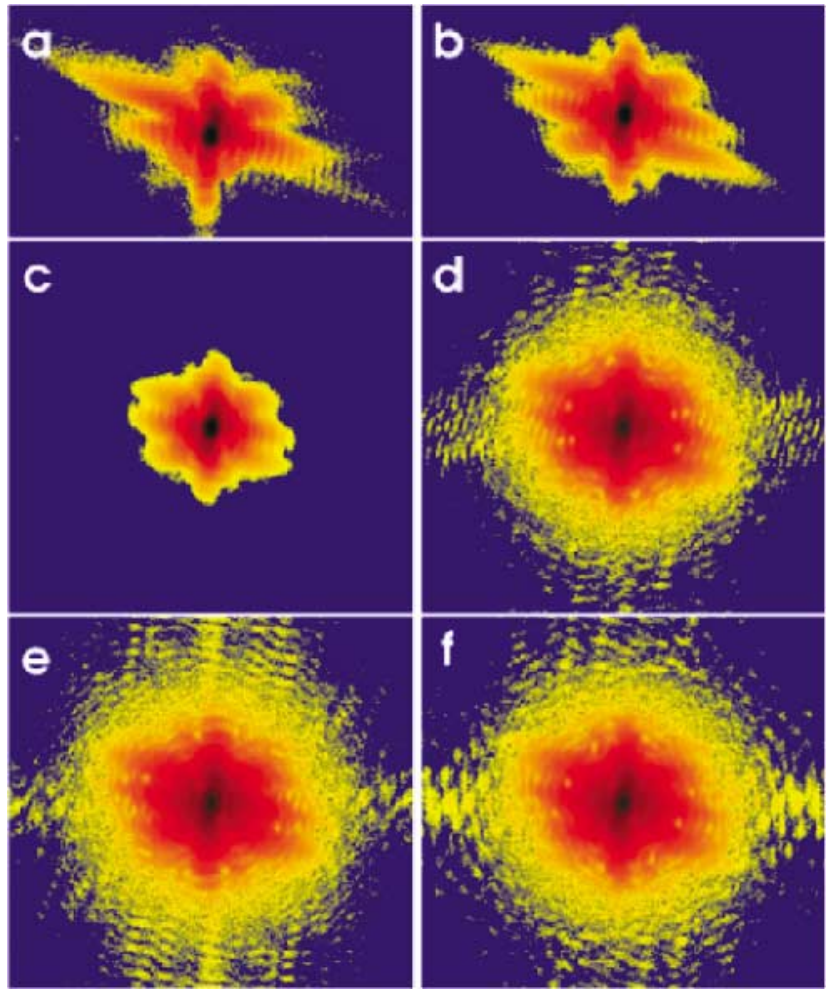

FIG. 2 (color). Stages of reconstruction of the diffraction data. (a) Coherent $\mathrm{x}$-ray diffraction pattern surrounding the specular (111) Bragg reflection of a $1 \mu \mathrm{m}$ gold nanocrystal, measured without a beamstop. (b) Symmetrized data. (c) Data filtered by multiplication by a circular Gaussian function. (d)-(f) Calculated diffraction patterns obtained by the inversion algorithm; the three examples used different random numbers for the initial phases at each pixel. The color contour levels have the same logarithmic spacing in all panels. ter of the diffraction pattern by averaging it with a rotated copy, producing the symmetric pattern of Fig. 2(b). To reduce aliasing problems, we applied a Gaussian filter function with a radial half-width of 41 pixels, as shown in Fig. 2(c). "Aliasing" refers to the spillover of density from adjacent unit cells, when the continuous Fourier transform in Eq. (1) is replaced by a discrete one, which introduces artificial periodic boundaries [30]. We then inverted the data using the iterative GS approach [21], consisting of Fienup's "error reduction" (ER) [22] and "hybrid inputoutput" (HIO) $[22,23]$ methods in alternation with a few dozen cycles of each. On the first cycle of ER, the amplitude of the diffraction was taken from Fig. 2(c), while each pixel's phase was taken to be a random number. The pattern was then Fourier-transformed to a 2D real-space image. The complex image was made real by setting its phase to zero, made positive, then confined to the $(45 \times 35)$-pixel rectangular "support" region indicated in Fig. 3(c) by setting the amplitude to zero outside. This "density-modified" image was transformed back to reciprocal space and compared with the data to obtain a residual $\chi^{2}$. For the subsequent cycles, the phases were retained while the amplitudes were overwritten by the measured values. The HIO method is similar except that the realspace step combines some memory of the previous cycle as well [23] and a limited amount of density is tolerated outside the support [23]. The residual $\chi^{2}$ was found to drop rapidly during the cycles. Repeating the calculation with different random phases did not lead to an identical solution, but a narrow distribution of slightly different $\chi^{2}$
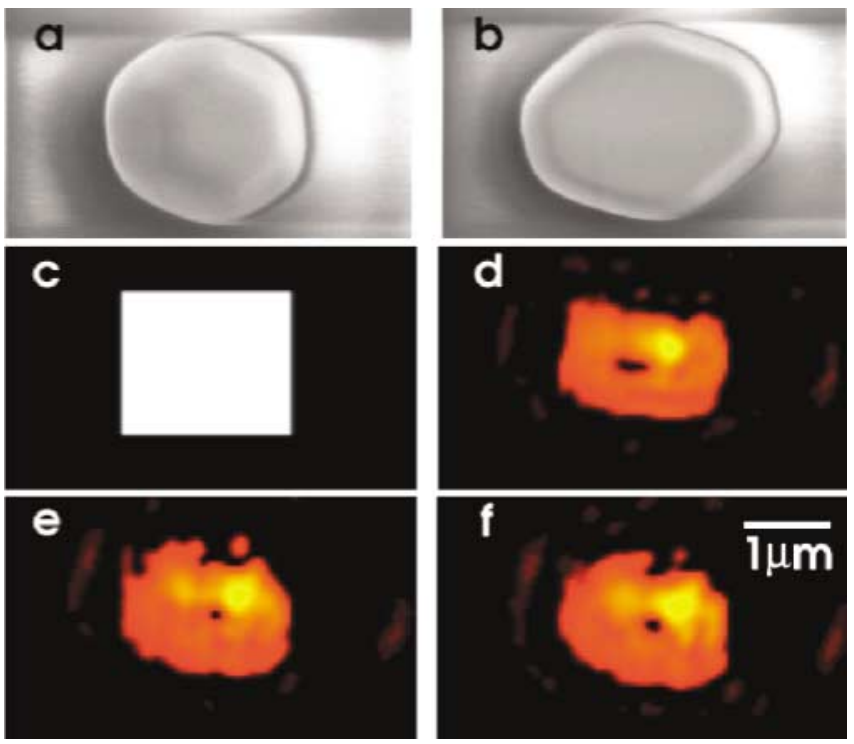

FIG. 3 (color). Reconstructed real-space projected images of the gold nanocrystals displayed on the same scale. (a),(b) SEM images of larger Au particles. (c) Size of the "support" constraint used in the inversion routines. (d)-(f) Real-space images obtained by inversion of the data in Fig. 2(c); the Fourier transforms of these images are the diffraction patterns shown in Figs. 2(d) $-2(\mathrm{f})$. 
values instead. Inspection of the images and the fitted diffraction patterns from several runs showed that the results were extremely similar to each other, as shown in Figs. 2 and 3(d) $-3(\mathrm{f})$.

For comparison purposes, we show in Figs. 3(a) and 3(b) SEM images of two of the larger gold nanocrystals from the sample, which show their polyhedral morphology. All of the projected images in Figs. 3(c)-3(f) have the same orientation as the data of Fig. 2 and all images are printed on the same scale. The aspect ratio of the inverted images has been restored by adjustment of the real-space pixel size, $\Delta x$, for the dimensions of the discrete fast Fourier transform (FFT) employed, $N_{\text {horiz }}=464$ and $N_{\text {vert }}=426$, according to

$$
2 \pi N=\Delta q \Delta x .
$$

The calculated diffraction patterns [Figs. 2(d)-2(f)] are obviously very similar to each other and give the same level of agreement with the data, $\chi^{2}=0.0038$, implying that the rms average pixel intensity differences are $6 \%$. The diffraction patterns are reproduced well with the most noticeable differences being at the extreme edges of the pattern where no intensity was recorded, but where the calculation produces small dense fringes. There is still some aliasing of the FFT visible in the calculated diffraction patterns, despite the Gaussian filter. The images themselves have the shapes and sizes expected on the basis of SEM, and flat edges can be clearly seen. The serrations may indicate the presence of a disordered skin, as noted above, but this will require further investigation. An unexpected feature, reproduced in every fit, is the appearance of an asymmetric "hot spot," significantly contracted from the crystal's boundary, which has about 3 times the apparent density of the remainder of the grain. Computational tests with simulated data have reproduced this feature and attributed it to partial coherence of the beam [27].

Coherent $x$-ray diffraction, coupled with the imaging capability we demonstrate, has considerable potential for understanding nanocrystalline materials. The penetrating power of a hard x-ray beam allows us to look below the surface of the material, yet retains the ability to separate the diffraction patterns from each individual grain. This paper has demonstrated inversion of $2 \mathrm{D}$ data to obtain a projection of the crystal shape, but 3D data should also be invertible by a sufficiently large calculation to the full shape in 3D. The $x$-ray penetration property means we can image the full volume of the grain with negligible effects of absorption. In principle, strain fields (projected onto the imaging $\mathbf{q}$ vector) should be imaged through the imaginary part of the real-space density, which will appear as a broken symmetry of the diffraction patterns [27]. The prospect of 3D real-space strain maps of the interior of materials is opened up by our result.

The UNICAT facility, located at the APS, is supported by the University of Illinois Materials Research Laboratory, Oak Ridge National Laboratory, the National Institute of Standards and Technologies, and UOP Research \& Development. The APS and the materials synthesis are supported by the U.S. Department of Energy (DOE) under Contracts No. W-31-109-ENG-38 and No. DEFG0298ER45439. This work was supported by the U.S. National Science Foundation under Contract No. DMR 98-76610.

*On leave from Institute of Crystallography RAS, Leninsky pr. 59, 117333 Moscow, Russia.

${ }^{\dagger}$ Present address: Bell Labs, Lucent Technologies, Murray Hill, NJ 07974.

[1] L. Pauling, The Nature of the Chemical Bond and the Structure of Molecules and Crystals (Cornell University, Ithaca, 1939).

[2] H. Schenk, Direct Methods of Solving Crystal Structures (Plenum, New York, 1991).

[3] B. C. Wang, Methods Enzymol. 115, 90-112 (1985).

[4] M. G. Rossmann and D. M. Blow, Acta Crystallogr. Sect. A 16, 39-45 (1963).

[5] G. Bricogne, Acta Crystallogr. Sect. A 40, 410-445 (1984).

[6] D. Sayre, Imaging Processes and Coherence in Physics, Springer Lecture Notes in Physics Vol. 112 (SpringerVerlag, Berlin, 1980), p. 229.

[7] J. Miao, D. Sayre, and H. N. Chapman, J. Opt. Soc. Am. A 15, 1662-1669 (1998).

[8] R. P. Millane, J. Opt. Soc. Am. A 7, 394-411 (1990).

[9] J. Miao, P. Charalambous, J. Kirz, and D. Sayre, Nature (London) 400, 342-344 (1999).

[10] D. Gabor, Nature (London) 161, 777 (1948).

[11] J. J. Barton, Phys. Rev. Lett. 61, 1356 (1988).

[12] G. R. Harp, D. K. Saldin, and B. P. Tonner, Phys. Rev. Lett. 65, 1012 (1990).

[13] J. Wilder et al., Phys. Rev. Lett. 86, 2337 (2001).

[14] T. Gog et al., Phys. Rev. Lett. 76, 3132 (1996).

[15] M. Tegze et al., Phys. Rev. Lett. 82, 4847 (1999).

[16] Q. Shen and R. Colella, Nature (London) 329, 232 (1987).

[17] Y. Yacoby et al., J. Phys. Condens. Matter 12, 3929 (2000).

[18] P. Cloetens et al., Appl. Phys. Lett. 75, 2912 (1999).

[19] M. Sutton et al., Nature (London) 352, 608-610 (1991).

[20] R. H. T. Bates, Optik (Stuttgart) 61, 247-262 (1982).

[21] R. W. Gerchberg and W. O. Saxton, Optik (Stuttgart) 35, 237-246 (1972).

[22] J. R. Fienup, Appl. Opt. 21, 2758-2369 (1982).

[23] R. P. Millane and W. J. Stroud, J. Opt. Soc. Am. A 14, 568-579 (1997).

[24] J. C. Heyraud and J. J. Métois, Acta Metall. 28, 1789-1797 (1980).

[25] M. Wortis, in Chemistry and Physics of Solid Surfaces VII, edited by R. Vanselow and R. Howe (Springer-Verlag, Berlin, 1988), p. 367.

[26] M. Born and E. Wolf, Principles of Optics (Pergamon Press, Oxford, 1984).

[27] I. A. Vartanyants and I. K. Robinson (to be published).

[28] M. von Laue, Ann. Phys. (Leipzig) 26, 55-85 (1936).

[29] I. K. Robinson, Phys. Rev. B 33, 3830 (1986).

[30] W. H. Press et al., Numerical Recipes (Cambridge University, Cambridge, England, 1988). 